\title{
One-dimensional Wiener process with the properties of partial reflection and delay
}

\author{
Kopytko B.I. ${ }^{1}$, Shevchuk R.V. ${ }^{2}$
}

In this paper, we construct the two-parameter semigroup of operators associated with a certain one-dimensional inhomogeneous diffusion process and study its properties. We are interested in the process on the real line which can be described as follows. At the interior points of the half-lines separated by a point, the position of which depends on the time variable, this process coincides with the Wiener process given there and its behavior on the common boundary of these half-lines is determined by a kind of the conjugation condition of Feller-Wentzell's type. The conjugation condition we consider is local and contains only the first-order derivatives of the unknown function with respect to each of its variables.

The study of the problem is done using analytical methods. With such an approach, the problem of existence of the desired semigroup leads to the corresponding conjugation problem for a second order linear parabolic equation to which the above problem is reduced. Its classical solvability is obtained by the boundary integral equations method under the assumption that the initial function is bounded and continuous on the whole real line, the parameters characterizing the Feller-Wentzell conjugation condition are continuous functions of the time variable, and the curve defining the common boundary of the domains is determined by the function which is continuously differentiable and its derivative satisfies the Hölder condition with exponent less than $1 / 2$. group.

Key words and phrases: diffusion process, parabolic equation, simple-layer potential, Feller semi-

${ }^{1}$ Czestochowa University of Technology, 69 Dabrowskiego str., 42-201, Czestochowa, Poland

${ }^{2}$ Lviv Polytechnic National University, 12 Bandera str., 79013, Lviv, Ukraine

E-mail: bohdan.kopytko@im.pcz.pl (Kopytko B.I.), r.v.shevchuk@gmail.com (Shevchuk R.V.)

\section{Introduction}

Consider the strip

$$
S_{t}=\{(s, x): \quad 0 \leq s<t \leq T, x \in \mathbb{R}\}
$$

in the plane $\mathbb{R}^{2}$ ( $T$ fixed) and two domains

$$
S_{t}^{(1)}=\{(s, x): \quad 0 \leq s<t \leq T,-\infty<x<r(s)\}
$$

and

$$
S_{t}^{(2)}=\{(s, x): \quad 0 \leq s<t \leq T, r(s)<x<\infty\}
$$

in it, where the function $x=r(s)$ belongs to the Hölder's class $H^{1+\alpha / 2}([0, T]), 0<\alpha<1$, (see [12, Ch. I, §1]). Denote by $\bar{S}_{t}, \bar{S}_{t}^{(1)}, \bar{S}_{t}^{(2)}$ the closures of $S_{t}, S_{t}^{(1)}, S_{t}^{(2)}$ respectively. Put

$$
D_{1 s}=(-\infty, r(s)), \quad D_{2 s}=(r(s), \infty), \quad \mathcal{C}=\{(s, r(s)): s \in[0, T]\}
$$

$\mathrm{y} \Delta \mathrm{K} 519.21$

2010 Mathematics Subject Classification: 60J60, 35K20. 
Our problem is to find a classical solution $u(s, x, t)$ of the equation

$$
\frac{\partial u}{\partial s}+\frac{1}{2} \frac{\partial^{2} u}{\partial x^{2}}=0, \quad(s, x) \in S_{t}^{(i)}, i=1,2
$$

which satisfies the "initial" condition

$$
\lim _{s \uparrow t} u(s, x, t)=\varphi(x), \quad x \in \mathbb{R},
$$

and two conjugation conditions

$$
\begin{gathered}
u(s, r(s)-0, t)=u(s, r(s)+0, t), \quad 0 \leq s \leq t \leq T \\
\sigma(s) \frac{\partial u}{\partial s}(s, r(s), t)+q_{1}(s) \frac{\partial u}{\partial x}(s, r(s)-0, t)-q_{2}(s) \frac{\partial u}{\partial x}(s, r(s)+0, t)=0, \quad 0 \leq s<t \leq T .
\end{gathered}
$$

The initial function $\varphi$ in (2) belongs to the space of bounded and continuous functions, that will be denoted by $C_{b}(\mathbb{R})$. The norm in this space is defined by $\|\varphi\|=\sup _{x \in \mathbb{R}}|\varphi(x)|$. The coefficients $\sigma, q_{i}, i=1,2$, in (4) are continuous on $[0, T]$ and

$$
\sigma(s)>0, \quad q_{1}(s) \geq 0, \quad q_{2}(s) \geq 0 \quad s \in[0, T]
$$

$u(s, r(s)-0, t)\left(\frac{\partial u}{\partial x}(s, r(s)-0, t)\right)$ and $u(s, r(s)+0, t)\left(\frac{\partial u}{\partial x}(s, r(s)+0, t)\right)$ denote the limits of the function $u(s, x, t)\left(\frac{\partial u}{\partial x}(s, x, t)\right)$ at $(s, r(s))$ as the point $(s, x)$ tends to $(s, r(s))$ from the side of the domains $S_{t}^{(1)}$ and $S_{t}^{(2)}$ respectively.

The problem (1)-(4) appears, in particular, in the theory of stochastic processes while studying the diffusion processes with boundary conditions. Recall that the general form of boundary conditions for one-dimensional diffusion processes was established by W. Feller [4] and A.D. Wentzell [20]. They proved the assertions from which it follows that if $\left\{T_{t}, t \geq 0\right\}$ is Feller semigroup in $C\left[r_{1}, r_{2}\right],-\infty<r_{1}<r_{2}<\infty$, and its generator $A$ is the restriction of $\left(L, C^{2}\left[r_{1}, r_{2}\right]\right)$, where $L$ is a second order ordinary differential operator, then functions from $\mathcal{D}_{A} \subset C^{2}\left[r_{1}, r_{2}\right]$ must satisfy boundary conditions which, generally speaking, have nonlocal character. These boundary conditions contain the value of the function and its first-order derivatives with respect to the time variable and with respect to the space variable at boundary points $r_{1}, r_{2}$, as well as the integral over $\left[r_{1}, r_{2}\right]$ with respect to some nonnegative measure which, furthermore, can be infinite.

The solving of parabolic problems of such kind (see $[9,11])$ is one of several ways to describe the diffusion process by given Feller-Wentzell boundary condition. Other approaches are reflected in many papers, see, e.g., $[4,13,18,20,21]$, where there are presented results of application of the analytical approach to description of the mentioned class of homogeneous Markov processes based on methods of the semigroup theory and functional analysis in relation to the elliptic boundary value problems, and $[1,3,6,14,16,17,19]$, which partially give the development of methods of stochastic analysis for the construction of such type of processes (see also the references given there). 
In the paper, we prove that there exists a unique solution $u(s, x, t)$ of $(1)-(4)$ and that the family of operators $T_{s t} \varphi(x) \equiv u(s, x, t)$ is the two-parameter Feller semigroup associated with an inhomogeneous diffusion process on the line $\mathbb{R}$. The trajectories of this process in $\bar{S}_{T} \backslash \mathcal{C}$ can be treated as the trajectories of the Wiener process generated by the operator $L \equiv \frac{1}{2} \frac{\partial^{2} u}{\partial x^{2}}$ and at the points of curve $\mathcal{C}$ its behavior is described by the conjugation condition (4). The condition (4) represents one of kinds of the general Feller-Wentzell conjugation condition (see $[4,13,20])$, which includes only the terms corresponding to the delay and partial reflection of the process at the moving point $x=r(s)$.

Note that the scheme, which is used to solve the problem (1)-(4), is partially presented in [11], where the same conjugation problem is considered for backward Kolmogotov equation with discontinuous coefficients and for the case when the condition (4) contains the nonlocal term of the integral type, but does not contain the term with derivative of the function with respect to the time variable. Note also that similar problems (with different variants of FellerWentzell conjugation condition) were studied in our earlier papers for the cases when $S_{t}^{(i)}$ are finite [10] or semi-infinite [9] rectangular domains.

The rest of this paper is organized as follows. Section 2 is devoted to the proof of the existence and uniqueness theorem for the conjugation problem (1)-(4). In Section 3, using the solution of this problem, we construct the two-parameter Feller semigroup on $C_{b}(\mathbb{R})$ and prove that this semigroup describes the ordinary diffusion process on $\mathbb{R}$, mentioned above.

\section{Existence and uniqueness}

The solution of the problem (1)-(4) will be constructed using the heat potentials generated by the fundamental solution $\Gamma(s, x, t, y), 0 \leq s<t \leq T, x, y \in \mathbb{R}$, of the equation (1):

$$
\Gamma(s, x, t, y)=\frac{1}{\sqrt{2 \pi(t-s)}} \exp \left\{-\frac{(y-x)^{2}}{2(t-s)}\right\} .
$$

The fact that $\Gamma$ is the fundamental solution of the equation (1) means that the function $\Gamma(s, x, t, y)$ satisfies this equation in $(s, x) \in[0, t) \times \mathbb{R}$ for fixed $(t, y)$ and

$$
\lim _{s \uparrow t} \int_{\mathbb{R}} \Gamma(s, x, t, y) \varphi(y) d y=\varphi(x), \quad x \in \mathbb{R},
$$

for any bounded continuous function $\varphi(x)$.

We note, among other properties of the fundamental solution $\Gamma$, that the function $\Gamma(s, x, t, y)$ is nonnegative and, for all $0 \leq s<t \leq T, x, y \in \mathbb{R}$,

$$
\left|D_{s}^{r} D_{x}^{p} \Gamma(s, x, t, y)\right| \leq C(t-s)^{-\frac{1+2 r+p}{2}} \exp \left\{-c \frac{(y-x)^{2}}{t-s}\right\}
$$

where $r$ and $p$ are any nonnegative integers, $C$ and $c$ are positive constants (in what follows, various positive constants depending on the data of the problem (1)-(4) will be denoted by $C$ or $c$ without specifying their values); $D_{s}^{r}$ is the partial derivative with respect to $s$ of order $r, D_{x}^{p}$ is the partial derivative with respect to $x$ of order $p$ (see [12, Ch.IV]). 
Note also that, for all $s \in[0, t), x \in \mathbb{R}$,

$$
\begin{aligned}
& \int_{\mathbb{R}} \Gamma(s, x, t, y) d y=1, \\
& \int_{\mathbb{R}} \Gamma(s, x, t, y)(y-x) d y=0, \\
& \int_{\mathbb{R}} \Gamma(s, x, t, y)(y-x)^{2} d y=t-s, \\
& \int_{\mathbb{R}} \Gamma(s, x, t, y)(y-x)^{4} d y=3(t-s)^{2} .
\end{aligned}
$$

Given the fundamental solution $\Gamma$, we define two heat potentials, which will be used to solve the problem (1)-(4), namely the Poisson potential

$$
u_{0}(s, x, t)=\int_{\mathbb{R}} \Gamma(s, x, t, y) \varphi(y) d y, \quad 0 \leq s<t \leq T, x \in \mathbb{R},
$$

and the simple-layer potential

$$
u_{1}(s, x, t)=\int_{s}^{t} \Gamma(s, x, \delta, r(\delta)) V(\delta, t) d \delta,
$$

where $\varphi(y), y \in \mathbb{R}$, and $V(\delta, t), \delta \in[0, t)$, are given functions.

If we assume that $\varphi \in C_{b}(\mathbb{R})$ and $V$ is bounded measurable for $\delta \in[0, t]$, then the functions $u_{0}$ and $u_{1}$ are continuous for $(s, x) \in[0, t] \times \mathbb{R}$, satisfy the equation (1) in the domains $(s, x) \in[0, t] \times \mathbb{R}$ and $(s, x) \in S_{t}^{(1)} \cup S_{t}^{(2)}$ respectively, and the initial conditions

$$
\lim _{s \uparrow t} u_{0}(s, x, t)=\varphi(x), \quad \lim _{s \uparrow t} u_{1}(s, x, t)=0, \quad x \in \mathbb{R} .
$$

Furthermore, for all $0 \leq s<t \leq T$ and $x \in \mathbb{R}$,

$$
\left|D_{s}^{r} D_{x}^{p} u_{0}(s, x, t)\right| \leq C(t-s)^{-\frac{2 r+p}{2}}\|\varphi\|,
$$

where $r$ and $p$ are any nonnegative integers.

An important property of the function $u_{1}$ is described by the so-called theorem on the jump of conormal derivative of a parabolic simple-layer potential (see [12, Ch.IV, §15], [15, Ch.XXII, $\S 8])$. Regarding the potential $u_{1}(s, x, t)$ in (13), this theorem asserts that if the function $V(\delta, t)$ is continuous in $0 \leq \delta<t \leq T$ and satisfies the inequality

$$
|V(\delta, t)| \leq C(t-\delta)^{-\mu}, \quad \mu<1
$$

in this domain, then for every point $x=r(s), s \in[0, t)$,

$$
\lim _{x \rightarrow r(s) \pm 0} \frac{\partial u_{1}}{\partial x}(s, x, t)=\mp V(s, t)+\int_{s}^{t} \frac{\partial \Gamma}{\partial x}(s, r(s), \delta, r(\delta)) V(\delta, t) d \delta .
$$

The integral in the right-hand side of (16) is called the direct value of conormal derivative of simple-layer potential. Its existence follows from the inequality

$$
\left|\frac{\partial \Gamma}{\partial x}(s, r(s), \delta, r(\delta))\right| \leq C(\delta-s)^{-\frac{1}{2}}, \quad 0 \leq s<\delta \leq T, i=1,2 .
$$


Theorem 1. Let the function $r(s), s \in[0, T]$, belongs to the Hölder's class $H^{1+\alpha / 2}([0, T])$, $0<\alpha<1$. Assume also that the functions $\sigma, q_{1}, q_{2}$ are continuous on $[0, T]$ and for them (5) holds. Then for any $\varphi \in C_{b}(\mathbb{R})$ the problem (1)-(4) has a unique solution which is continuous in $\bar{S}_{t}$.

Proof. We look for a solution $u(s, x, t)$ of the problem (1)-(4) of the form

$$
u(s, x, t)=u_{0}(s, x, t)+u_{1}(s, x, t), \quad(s, x) \in \bar{S}_{t},
$$

where the functions $u_{0}$ and $u_{1}$ are defined by the formulas (12) and (13) respectively, in which $\varphi$ is the initial function in (2) and $V$ is the unknown function to be found.

Suppose a priori that the unknown density $V(\delta, t), i=1,2$, is continuous in the domain $0 \leq \delta<t \leq T$ and satisfies (15) in this domain. Using the conjugation conditions (3), (4), in view of (16), we obtain the following relation

$$
\frac{\partial u}{\partial s}(s, r(s), t)=\Phi_{0}(s, t), \quad 0 \leq s<t \leq T,
$$

where

$$
\begin{aligned}
& \Phi_{0}(s, t)=-q(s) V(s, t)+\gamma(s) \int_{s}^{t} \frac{\partial \Gamma}{\partial x}(s, r(s), \delta, r(\delta)) V(\delta, t) d \delta+\gamma(s) \frac{\partial u_{0}}{\partial x}(s, r(s), t), \\
& q(s)=\frac{q_{2}(s)+q_{1}(s)}{\sigma(s)}, \quad \gamma(s)=\frac{q_{2}(s)-q_{1}(s)}{\sigma(s)}+r^{\prime}(s) .
\end{aligned}
$$

The relation (18) can be considered as the autonomous differential equation for the function $u(s, r(s), t)$ in the domain $s \in[0, t)$. Taking into account the initial condition

$$
\lim _{s \uparrow t} u(s, r(s), t)=\varphi(r(t)),
$$

we can write the solution of this equation in the form

$$
u(s, r(s), t)=\varphi(r(t))-\int_{s}^{t} \Phi_{0}(\tau, t) d \tau .
$$

Thus, we have two different expressions for $u(s, r(s), t)$, namely the relation (17) with $x=r(s)$ and the relation (19). Equating their right-hand sides, we get the following integral equation for $V$

$$
\int_{s}^{t} K_{0}(s, \delta) V(\delta, t) d \delta=\psi_{0}(s, t)
$$

where

$$
\begin{aligned}
& K_{0}(s, \delta)=\Gamma(s, r(s), \delta, r(\delta))-q(\delta)+\int_{s}^{t} \gamma(\tau) \frac{\partial \Gamma}{\partial x}(\tau, r(\tau), \delta, r(\delta)) d \tau, \\
& \psi_{0}(s, t)=\varphi(r(t))-u_{0}(s, r(s), t)-\int_{s}^{t} \gamma(\tau) \frac{\partial u_{0}}{\partial x}(\tau, r(\tau), t) d \tau .
\end{aligned}
$$


Note that (20) is the Volterra integral equation of the first kind and that the function $\psi_{0}$ in the right-hand side of this equation is continuously differentiable in $s, 0 \leq s<t \leq T$. Using the Holmgren transform (see [7]), we reduce this equation to the equivalent Volterra integral equation of the second kind. To do this, we introduce the integro-differential operator $\mathcal{E}$ which acts by the rule

$$
\mathcal{E}(s, t) f=\sqrt{\frac{2}{\pi}} \frac{\partial}{\partial s} \int_{s}^{t}(\rho-s)^{-\frac{1}{2}} f(\rho, t) d \rho, \quad 0 \leq s<t \leq T,
$$

and apply it to the both sides of (20).

Consider first the application of the operator $\mathcal{E}$ to the right-hand side of (20), i.e. to the function $\psi_{0}(s, t)$. In view of (14), for the function $\psi_{0}(s, t)$ and its derivative

$$
\frac{\partial \psi_{0}}{\partial s}(s, t)=-\frac{\partial u_{0}}{\partial s}(s, r(s), t)+\gamma(s) \frac{\partial u_{0}}{\partial x}(s, r(s), t),
$$

we find the following estimates (here $0 \leq s<t \leq T$ ):

$$
\begin{gathered}
\left|\psi_{0}(s, t)\right| \leq C\|\varphi\|, \\
\left|\frac{\partial \psi_{0}}{\partial s}(s, t)\right| \leq C\|\varphi\|(t-s)^{-1} .
\end{gathered}
$$

Since $\psi_{0}(s, t)$ is continuously differentiable in $s(s \in[0, t))$, we have

$$
\psi(s, t) \equiv \mathcal{E}(s, t) \psi_{0}=-\sqrt{\frac{2}{\pi}}(t-s)^{-\frac{1}{2}} \psi_{0}(s, t)+\frac{1}{\sqrt{2 \pi}} \int_{s}^{t}(\rho-s)^{-\frac{3}{2}}\left[\psi_{0}(\rho, t)-\psi_{0}(s, t)\right] d \rho .
$$

Denote by $\psi^{(1)}(s, t)$ and $\psi^{(2)}(s, t)$ the first and second terms in (23), respectively. It follows from (21) that

$$
\left|\psi^{(1)}(s, t)\right| \leq C\|\varphi\|(t-s)^{-\frac{1}{2}}, \quad 0 \leq s<t \leq T .
$$

To estimate $\psi^{(2)}(s, t)$, write it in the form

$$
\begin{aligned}
\psi^{(2)}(s, t) & =\frac{1}{\sqrt{2 \pi}} \int_{s}^{\frac{s+t}{2}}(\rho-s)^{-\frac{3}{2}}\left[\psi_{0}(\rho, t)-\psi_{0}(s, t)\right] d \rho \\
& +\frac{1}{\sqrt{2 \pi}} \int_{\frac{s+t}{2}}^{t}(\rho-s)^{-\frac{3}{2}}\left[\psi_{0}(\rho, t)-\psi_{0}(s, t)\right] d \rho \equiv I_{1}(s, t)+I_{2}(s, t) .
\end{aligned}
$$

Applying the mean value theorem to the difference $\psi_{0}(\rho, t)-\psi_{0}(s, t)$ in the first term in (25) and using (22), we see that for this term the inequality (24) holds. Moreover, from (21) it follows that the same estimate is true also for the second term in (25). Thus, $\psi^{(2)}(s, t)$ allows the estimate (24).

Having estimated $\psi^{(1)}(s, t)$ and $\psi^{(2)}(s, t)$, in view of $(23)$, we conclude that

$$
|\psi(s, t)| \leq C\|\varphi\|(t-s)^{-\frac{1}{2}}, \quad 0 \leq s<t \leq T .
$$


Consider now the application of the operator $\mathcal{E}$ to the left-hand side of (20). After simple transformations, we get

$$
\sqrt{\frac{2}{\pi}} \frac{\partial}{\partial s} \int_{s}^{t}(\rho-s)^{-\frac{1}{2}} d \rho \int_{\rho}^{t} K_{0}(\rho, \delta) V(\delta, t) d \delta=-V(s, t)+\int_{\rho}^{t} N(s, \delta) V(\delta, t) d \delta
$$

where

$$
\begin{aligned}
N(s, \delta)= & \frac{1}{\pi} \frac{\partial}{\partial s} \int_{s}^{\delta}(\delta-\rho)^{-\frac{1}{2}}(\rho-s)^{-\frac{1}{2}}\left[\exp \left\{-\frac{(r(\delta)-r(\rho))^{2}}{2(\delta-\rho)}\right\}-1\right] d \rho \\
& +\sqrt{\frac{2}{\pi}} q(\delta)(\delta-s)^{-\frac{1}{2}}+\sqrt{\frac{2}{\pi}} \frac{\partial}{\partial s} \int_{s}^{\delta}(\rho-s)^{-\frac{1}{2}} d \rho \int_{\rho}^{\delta} \gamma(\tau) \frac{\partial \Gamma}{\partial x}(\tau, r(\tau), \delta, r(\delta)) d \tau .
\end{aligned}
$$

Using the considerations similar to those leading to (26), one can establish the following estimate

$$
|N(s, \delta)| \leq C(\delta-s)^{-\frac{1}{2}}, \quad 0 \leq s<\delta \leq t \leq T
$$

Thus, the application of the operator $\mathcal{E}$ to both sides of (20) gives the following Volterra integral equation of the second kind, which is equivalent to (20),

$$
V(s, t)=\int_{s}^{t} N(s, \delta) V(\delta, t) d \delta-\psi(s, t),
$$

where the function $\psi(s, t)$ is defined by the formula (23) and the kernel of this equation, i.e the function $N(s, \delta)$ is defined by (1). Furthermore, the estimates (26) and (27) hold. These estimates ensure the existence and uniqueness of the solution of (28) in the class of continuous functions in $0 \leq s<t \leq T$ which can be found by the method of successive approximations and for which the estimate (26) holds.

From the estimates (7) (with $r=p=0$ ) and (26) (with $\psi$ replaced by $V$ ), it follows that the simple-layer potential $u_{1}(s, x, t)$ in (17) is well-defined and for it the estimate

$$
\left|u_{1}(s, x, t)\right| \leq C\|\varphi\|, \quad(s, x) \in \bar{S}_{t},
$$

holds. It is clear (see (14)) that the same estimate is also true for the Poisson potential $u_{0}(s, x, t)$ in (12) and thus for the function $u(s, x, t)$ itself.

Concerning the "initial" condition (2), note that the function $u$ in (17) satisfies it, because

$$
\lim _{s \uparrow t} u_{0}(s, x, t)=\varphi(x), \quad \lim _{s \uparrow t} u_{1}(s, x, t)=0, \quad x \in \mathbb{R} .
$$

However, since the function $V(s, t)$ is "weakly" bounded by $C(t-s)^{-\frac{1}{2}}$, the second relation in (29) is not obvious and must be verified. To do this, we consider the function $V(s, t), s \in[0, t)$, and study its behavior in a neighborhood of the point $s=t$. Using the considerations similar to those leading to (26), one can prove the following assertion: for every $t \in(0, T]$ and $\varepsilon>0$ there exists $s_{0} \in[0, t)$ such that the inequality

$$
|V(s, t)| \leq \varepsilon C(t-s)^{-\frac{1}{2}}
$$


holds for all $s \in\left[s_{0}, t\right)$, where the constant $C$ is independent of $\varepsilon$.

Let $\varepsilon>0$ and choose $s_{0}$ such that (30) holds for all $s \in\left[s_{0}, t\right)$. Then, from (30) and (7), we deduce that

$$
\left|u_{1}(s, x, t)\right| \leq \varepsilon C \int_{s}^{t}(\tau-s)^{-\frac{1}{2}}(t-\tau)^{-\frac{1}{2}} d \tau=\varepsilon C \pi
$$

(the constant $C$ is independent of $\varepsilon$ ) for all $s \in\left[s_{0}, t\right)$ and $x \in \mathbb{R}$. Because of the arbitrariness of $\varepsilon$ this implies that $u_{1}(s, x, t)$ tends to zero if $s \uparrow t$ (for all $x \in \mathbb{R}$ ).

Thus, the function $u(s, x, t)\left((s, x) \in \bar{S}_{t}\right)$ of the form (17), where the density $V$ (in the expression for the simple-layer potential $u_{1}$ ) is defined as the solution of (28), is the desired solution of the conjugation problem (1)-(4).

In order to complete the proof of the theorem, it remains to establish the uniqueness of the solution of the problem (1)-(4). For this purpose, it suffices to note that the constructed function $u(s, x, t)$ in each of two domains $S_{t}^{(1)}, S_{t}^{(2)}$ can be treated as a unique solution of the following first boundary-value parabolic problem

$$
\begin{aligned}
& \frac{\partial u}{\partial s}+\frac{1}{2} \frac{\partial^{2} u}{\partial x^{2}}=0, \quad(s, x) \in S_{t}^{(i)}, i=1,2, \\
& \lim _{s \uparrow t} u(s, x, t)=\varphi(x), \quad x \in D_{i t}, i=1,2, \\
& u(s, r(s), t)=v(s, r(s), t)
\end{aligned}
$$

where the function $v$ is defined by the right-hand side of (19).

The proof of Theorem 1 is complete.

\section{Construction of the Feller semigroup}

Let the conditions of Theorem 1 hold. Define the two-parameter family of operators $T_{s t}$, $0 \leq s<t \leq T$, in $C_{b}(\mathbb{R})$ by the formula

$$
T_{s t} \varphi(x)=T_{s t}^{(0)} \varphi(x)+T_{s t}^{(1)} \varphi(x), \quad 0 \leq s<t \leq T, x \in \mathbb{R},
$$

where

$$
\begin{aligned}
& T_{s t}^{(0)} \varphi(x)=u_{0}(s, x, t)=\int_{\mathbb{R}} \Gamma(s, x, t, y) \varphi(y) d y, \\
& T_{s t}^{(1)} \varphi(x)=u_{1}(s, x, t)=\int_{s}^{t} \Gamma(s, x, \delta, r(\delta)) V(\delta, t) d \delta,
\end{aligned}
$$

and the function $V$ is the solution of the second kind Volterra integral equation (28).

The presence of the integral representation for the family of operators $T_{s t}, 0 \leq s<t \leq T$, allows us to verify easily the following conditions:

1) if $\varphi_{n} \in C_{b}(\mathbb{R}), n=1,2, \ldots, \sup _{n}\left\|\varphi_{n}\right\|<\infty$ and for all $x \in \mathbb{R}, \lim _{n \rightarrow \infty} \varphi_{n}(x)=\varphi(x)$, where $\varphi \in C_{b}(\mathbb{R})$, then for all $(s, x) \in \bar{S}_{t}$,

$$
\lim _{n \rightarrow \infty} T_{s t} \varphi_{n}(x)=T_{s t} \varphi(x)
$$


2) $T_{s t} \varphi(x) \geq 0$ for all $(s, x) \in \bar{S}_{t}$ if $\varphi \in C_{b}(\mathbb{R})$ and $\varphi(x) \geq 0$ for all $x \in \mathbb{R}$;

3) the operators $T_{s t}$ are contractive, i.e.

$$
\left\|T_{s t} \varphi\right\| \leq\|\varphi\|
$$

for any $\varphi \in C_{b}(\mathbb{R})$;

4) for all $0 \leq s \leq \tau \leq t \leq T$,

$$
T_{s t}=T_{s \tau} T_{\tau t}
$$

Let us get down to proving these properties. The first one follows from the Lebesgue bounded convergence theorem and the relation

$$
\lim _{n \rightarrow \infty} V\left(s, t, \varphi_{n}\right)=V(s, t, \varphi), \quad s \in[0, t),
$$

which holds for the solution of the integral equation (28).

We now prove that the operators $T_{s t}$ do not increase the norm of element. In view of the property 1), it suffices to show that $T_{s t} \varphi(x) \geq 0$ for all $(s, x) \in \bar{S}_{t}$ in case the nonnegative function $\varphi \in C_{b}(\mathbb{R})$ has a compact support. Suppose that $\varphi$ is just that. Suppose also that

$$
\min _{s \in[0, t], x \in \mathbb{R}} T_{s t} \varphi(x)=u_{*}<0 .
$$

Note that the function $T_{s t} \varphi(x)$ satisfies the equation (1) in $(s, x) \in S_{t}^{(i)}, i=1,2$, and that $T_{s t} \varphi(x)$ tends to zero as $|x| \rightarrow \infty$. From this and from the minimum principle for parabolic equations it follows that there exists $s_{0} \in[0, t)$ for which $T_{s_{0}} \varphi\left(r\left(s_{0}\right)\right)=u_{*}$. Then, at point $\left.\left(s_{0}, r\left(s_{0}\right)\right)\right)$, the following relations hold:

$$
\frac{\partial}{\partial s} T_{s_{0} t} \varphi\left(r\left(s_{0}\right)\right)=0, \quad \frac{\partial}{\partial x} T_{s_{0} t} \varphi\left(r\left(s_{0}\right)-0\right) \leq 0, \quad \frac{\partial}{\partial x} T_{s_{0} t} \varphi\left(r\left(s_{0}\right)+0\right) \geq 0 .
$$

Moreover, it follows from Theorem 1 in [8] (cf. Theorem 14 in [5, Ch.II, §4]) that in the last two estimates, the equal signs should be excluded. Hence, at point $\left(s_{0}, r\left(s_{0}\right)\right)$, the conjugation condition (4) cannot hold. The contradiction we arrived at indicates that $u_{*} \geq 0$. This completes the proof.

The property 3) of the operator $T_{s t}$ is an easy consequence of the property 2 ) and the obvious relation $T_{s t} 1 \equiv 1$.

The last property, which means that the family of operators $T_{s t}$ is a two-parameter semigroup, follows from the assertion of Theorem 1 on uniqueness of the solution of the problem (1)-(4). Indeed, to find $u(s, x, t)$ when $u(t, x, t)=\varphi(x)$, one can do the following: solve the equation in the time interval $[\tau, t]$ and then solve it in the time interval $[s, \tau]$ starting with $u(\tau, x, t)$ which was obtained; in other words, $T_{s t} \varphi=T_{s \tau}\left(T_{\tau t} \varphi\right), \varphi \in C_{b}(\mathbb{R})$, or $T_{s t}=T_{s \tau} T_{\tau t}$.

From the properties 1)-4) if follows that (see, e.g., [2, Ch.II, \$1]) the family of operators $T_{\text {st }}$, $0 \leq s \leq t \leq T$, is a semigroup associated with some inhomogeneous Feller process on $\mathbb{R}$. If we denote by $P(s, x, t, d y)$ its transition function, then $T_{s t} \varphi(x)$ can be represented as

$$
T_{s t} \varphi(x)=\int_{\mathbb{R}} \varphi(y) P(s, x, t, d y) .
$$


Finally, taking into account (8)-(11), we find by direct calculation that

$$
\begin{gathered}
\sup _{x \in \mathbb{R}} \int_{\mathbb{R}}|y-x|^{4} P(s, x, t, d y) \leq C(t-s)^{2}, \quad 0 \leq s<t \leq T, \\
a(s, x) \equiv \lim _{\Delta s \downarrow 0} \frac{1}{\Delta s} \int_{\mathbb{R}}(y-x) P(s, x, s+\Delta s, d y) \\
\quad= \begin{cases}0 & \text { for } \quad(s, x) \in S_{t}^{(i)}, i=1,2, \\
\gamma(s) & \text { for } \quad x=r(s),\end{cases} \\
b(s, x) \equiv \lim _{\Delta s \downarrow 0} \frac{1}{\Delta s} \int_{\mathbb{R}}(y-x)^{2} P(s, x, s+\Delta s, d y)=b(s, x) \\
= \begin{cases}1 & \text { for } \quad(s, x) \in S_{t}^{(i)}, i=1,2, \\
0 & \text { for } \quad x=r(s) .\end{cases}
\end{gathered}
$$

The inequality (32) shows that the sample paths of the constructed process are continuous. The relations (33) and (34) mean that this process can be treated as the ordinary diffusion process with the drift coefficient $a(s, x)$ and the diffusion coefficient $b(s, x)$.

Thus, the following theorem is a conclusion of the second part of our research.

Theorem 2. Let the conditions of Theorem 1 be satisfied. Then the two-parameter family of the operators $T_{s t}, 0 \leq s<t \leq T$, defined by (31) is the semigroup associated with the inhomogeneous diffusion process on $\mathbb{R}$, the transition function $P(s, x, t, d y)$ of which satisfies the relations (33), (34).

\section{References}

[1] Anulova S.V. Diffusion processes: discontinuous coefficients, degenerate diffusion, randomized drift. Dokl. Akad. Nauk 1981, 260 (5), 1036-1040. (in Russian)

[2] Dynkin E.B. Markov processes. Gosudarstv. Izdat. Fiz.-Mat. Lit., Moscow, 1963. (in Russian)

[3] Engelbert H.J., Schmidt W. Strong Markov continuous local martingales and solutions of one-dimensional stochastic differential equations. III. Math. Nachr. 1991, 151 (1), 149-197. doi:10.1002/mana.19911510111

[4] Feller W. The parabolic differential equations and the associated semi-groups of transformations. Ann. of Math.(2) 1952, 55 (3), 468-519. doi:10.2307/1969644

[5] Friedman A. Partial differential equations of parabolic type. Prentice-Hall, Englewood Cliffs, New Jersey, 1964.

[6] Ikeda N., Watanabe S. Stochastic differential equations and diffusion processes. North-Holland Mathematical Library 24, North-Holland Publishing Co., Amsterdam-New York, Kodansha, Ltd., Tokyo, 1981.

[7] Kamynin L.I. A boundary value problem in the theory of heat conduction with a nonclassical boundary condition. Zh. Vychisl. Mat. Mat. Fiz. 1964, 4 (6), 1006-1024.

[8] Kamynin L.I., Khimchenko B.N On applications of the maximum principle to parabolic equations of second order. Dokl. Akad. Nauk 1972, 204 (3), 529-532. (in Russian)

[9] Kopytko B.I., Shevchuk R.V. On pasting together two inhomogeneous diffusion processes on a line with the general Feller-Wentzell conjugation condition. Theory Stoch. Process. 2011, 17 (2), 55-70.

[10] Kopytko B.I., Shevchuk R.V. One-dimensional diffusion processes in bounded domains with boundary conditions and conjugation condition of Feller-Wentzell. Bukovinian Math. Journal 2013, 1 (1-2), 77-85. 
[11] Kopytko B.I., Shevchuk R.V. One-dimensional diffusion processes with moving membrane: partial reflection in combination with jump-like exit of process from membrane. Electron. J. Probab. 2020, 25 (41), 1-21. doi:10.1214/20EJP443

[12] Ladyzhenskaya O.A., Solonnikov V.A., Ural'tseva N.N. Linear and Quasilinear Equations of Parabolic Type. Nauka, Moscow, 1967. (in Russian)

[13] Langer H., Schenk W. Knotting of one-dimensional Feller processes. Math. Nachr. 1983, 113 (1), $151-161$. doi:10.1002/mana.19831130115

[14] Pilipenko A.Yu. On the Skorokhod mapping for equations with reflection and possible jump-like exit from a boundary. Ukrainian Math. J. 2012, 63 (9), 1415-1432. doi:10.1007/s11253-012-0588-2

[15] Pogorzelski W. Integral equations and their applications. Tom IV. PWN-Polish Scientific Publishers, Warsaw, 1970. (in Polish)

[16] Portenko M.I. Diffusion Processes in Media with Membranes. Proceedings of the Institute of Mathematics of the National Academy of Sciences of the Ukraine, Kyiv, 1995. (in Ukrainian)

[17] Skorokhod A.V. Stochastic equations for diffusion processes in a bounded region. Theory Probab. Appl. 1961, 6 (3), 264-274. doi: 10.1137/1106035

[18] Skubachevskii A.L. Nonlocal elliptic problems and multidimensional diffusion processes. Russ. J. Math. Phys. 1995, 3 (3), 327-360.

[19] Walsh J.B. A diffusion with a discontinuous local time. Astérisque 1978, 52-53, 37-45.

[20] Wentzell A.D. Semigroups of Operators Corresponding to a Generalized Differential Operatorof Second Order. Dokl. Akad. Nauk 1956, 111 (2), 269-272. (in Russian)

[21] Venttsel A.D. On boundary conditions for multi-dimensional diffusion processes. Theory Probab. Appl. 1959, 4 (2), 164-177. doi: 10.1137/1104014

Received 14.03.2021

Revised 22.03.2021

Копитко Б.І., Шевчук Р.В. Одновимірний вінерівський процес з властивостями иасткового відбитms $і$ затримки // Карпатські матем. публ. - 2021. - Т.13, №2. - С. 534-544.

Метою статті $є$ побудова та дослідження властивостей двопараметричної напівгрупи Феллера, якій відповідає неоднорідний дифузійний процес на прямій такий, що у внутрішніх точках півпрямих, розділених між собою точкою, положення якої на прямій залежить від часової змінної, він збігається із заданим там вінерівським процесом, а його поведінка на спільній межі областей визначається одним із варіантів умови спряження типу Феллера-Вентцеля. У розглядуваному нами випадку ця умова є локальною і містить в якості складових лише похідні першого порядку невідомої функції по кожній змінній.

Аля розв' язання цієї задачі в роботі застосовано аналітичні методи. За такого підходу питання про існування шуканої напівгрупи зводиться до розв'язання відповідної задачі спряження Аля лінійного параболічного рівняння другого порядку, до якої редукується вихідна проблема. Її класичну розв'язність отримано в роботі методом граничних інтегральних рівнянь за припущення, що початкова функція є обмеженою і неперервною на всій числовій прямій, параметри, які характеризують умову спряження Феллера-Вентцеля $є$ неперервними функціями часової змінної, а крива, що визначає спільну межу областей, задається функцією, що $є$ неперервно диференційовною, до того ж ії похідна задовольняє умову Гельдера з показником меншим, ніж $1 / 2$.

Ключові слова і фрази: дифузійний процес, параболічне рівняння, потенщіал простого шару, напівгрупа Феллера. 